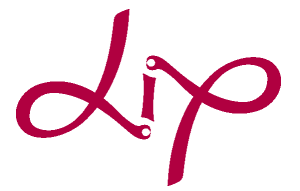

Laboratoire de l'Informatique du Parallélisme

École Normale Supérieure de Lyon

Unité Mixte de Recherche CNRS-INRIA-ENS LYON-UCBL n ${ }^{\circ} 5668$

\title{
Multi-phase On-chip Traffic Generation Environment
}

Antoine Scherrer,

Antoine Fraboulet,

Tanguy Risset

June 2006

École Normale Supérieure de Lyon

46 Allée d'Italie, 69364 Lyon Cedex 07, France

Téléphone : $+33(0) 4.72 .72 .80 .37$

Télécopieur : $+33(0) 4.72 .72 .80 .80$

Adresse électronique : lip@ens-lyon.fr CENTRE National
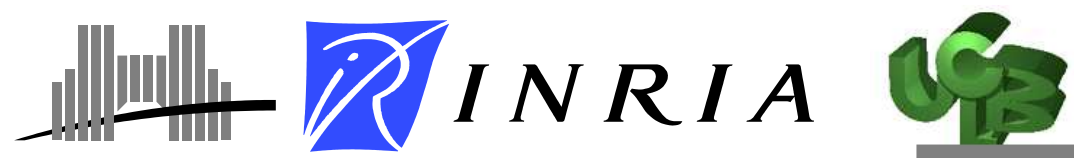


\title{
Multi-phase On-chip Traffic Generation Environment
}

\author{
Antoine Scherrer, Antoine Fraboulet, Tanguy Risset
}

June 2006

\begin{abstract}
In this report, we present a cycle-accurate multi-phase on-chip traffic generation and simulation environment. This environment provides deterministic traffic generation (traces replay) as well as stochastic traffic generation based on statistical analysis of a reference trace. We show that our traffic generators emulate precisely the behavior of processors or IP and that our environment is a versatile tool for network-on-chip prototyping. Simulations are performed in a SystemC-based simulation environment with a mesh network-on-chip
\end{abstract}

Keywords: On-Chip Traffic, Multi-Phase Behavior, Stochastic Analysis

\section{Résumé}

Dans ce rapport, nous présentons un environnement de génération de trafic sur puce précis au cycle. Cet environnement peut soit rejouer une trace de trafic enregistrée, soit générer du trafic stochastique ajuster sur la base d'une analyse statistique d'une trace donnée. Nous montrons dans ce rapport que le trafic produit par notre générateur est assez proche de celui produit par les processeurs ou IP que nous cherchons à remplacer. Nous validons cette approche par des simulation effectuées dans un environement utilisant SystemC.

Mots-clés: Traffic sur puce, analyse stochastique, comportement multi-phase 


\section{Contents}

1 Introduction $r$

2 On-chip traffic generation $\quad 3$

2.1 Deterministic traffic generation . . . . . . . . . . . . . . . . . . . . . . . . . . . . . .

2.2 Stochastic traffic generation . . . . . . . . . . . . . . . . . . . . . . . . . . . . . . . . . . . . . . . . . .

2.3 Traffic models . . . . . . . . . . . . . . . . . . . . . . . . . . . . . . . . . . . . . . . . . . .

2.3.1 First order statistics . . . . . . . . . . . . . . . . . . . 4

2.3.2 Second order statistics . . . . . . . . . . . . . . . . . . 4

2.4 Phases in traffic . . . . . . . . . . . . . . . . . . . . 5

3 Multi-Phase Traffic Generator $\quad 5$

3.1 On-chip traffic modeling . . . . . . . . . . . . . . . . . . 5

3.2 Global methodology . . . . . . . . . . . . . . . . . . . . . . . . . . . . . . . . . . . . .

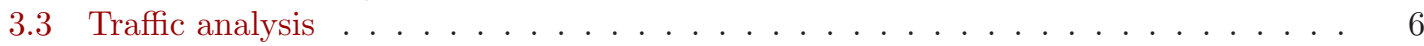

3.4 Traffic synthesis . . . . . . . . . . . . . . . . . . . . . . . . 7

3.5 Traffic generator key features . . . . . . . . . . . . . . . . . . 7

4 Simulation environment and set-up $\quad 8$

4.1 SocLib . . . . . . . . . . . . . . . . . . . . . . 8

4.2 Simulation flow . . . . . . . . . . . . . . . . . . . . 8

5 Experimental results $\quad 9$

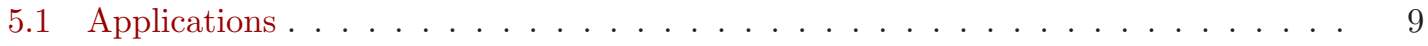

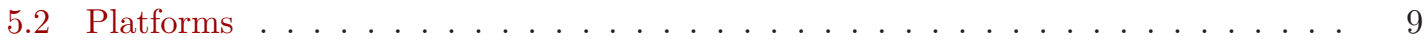

5.3 Design time setup . . . . . . . . . . . . . . . . . . . . . 10

5.4 Simulation time . . . . . . . . . . . . . . . . . . . . . . . . . . . . . . . . . . . . . 10

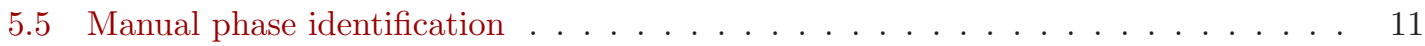

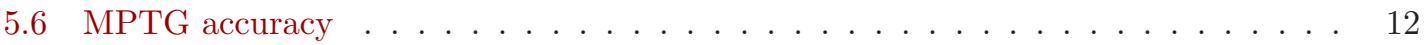

6 Conclusion $\quad 17$ 


\section{Introduction}

Systems on chip (SoC) are now commonly used in embedded systems for multimedia and telecommunication applications. Most of these SoC are composed of a single processor controlling various components (Intellectual Property: IP) connected all-together. The computing power required by emerging applications running on mobile terminals, such as video on mobile phone for instance, has pushed the development of a more complex SoC infrastructure, the so called multi-processor SoC (MPSoC) typically composed of a number of master components (processors or DMA for hardware accelerators) connected to a network on chip (NoC) or a hierarchy of busses.

The advent of networks-on-chip (NoC) has significantly increased the design complexity of such systems with some hard problems related to parallelism: non-determinism, memory and cache coherency, efficient workload distribution and network contention. Solving these problems during the short time available for design requires fundamental improvements in design methodologies. The most important shift is the setting of a refinement design methodology allowing designers to explore design space at various levels of precision. These levels, called transaction, bus-accurate, synthesizable, etc. allow the designer to check quickly that performances related to various metrics are achieved before writing the complete description of the system.

During design space exploration, simulation time is a major problem. There are two run-time behaviors very difficult to model at a high level: cache behavior and network contention. Precise simulation of these two behaviors can only be done with a low-level description of the components. This means hours (sometimes days) of simulation for a single execution or, as it is usually preferred, the use of extremely expensive hardware emulators.

Reducing simulation time can be achieved by a clever analysis of the behavior of the system during execution. We are interested in the simulation of on-chip network behavior and performance evaluation. Traffic generators (TG) are more and more used during SoC design for platform prototyping or performance evaluation. When using TGs, simulation time is decreased because the IP is not fully simulated. Simulation is also more flexible: $i$ ) the same generic TG can be used for a class of similar applications, $i i$ ) the designer does not need to integrate the complete IP in its simulation environment, which is useful in early stage of design if the IP is not fully specified or provided by a $3^{\text {rd }}$ party.

Most recent traffic generation methods use stochastic models. Statistical analysis and synthesis of on-chip traffic is difficult because this traffic usually presents complex statistical behavior: nonstationarity and long-range-dependence [17]. The precision of the simulation and the possibility of integrating the whole process in an automatic (or at least semi-automatic) framework are important parameters for evaluating the usefulness of a traffic generation environment.

In this paper, we present a new traffic simulation environment based on traffic generators that can be parameterized. Our TG gathers and extends different features presented individually in various recent works making it a very flexible tool for network-on-chip prototyping. It can run a deterministic traffic replay (as in [11]) or generate a stochastic traffic with first order (as in [9]) and second order (as in [17]) statistics fitted to a particular trace. An important feature of our TG is the fact that it can run a traffic divided into separate phases, each phase having different characteristics. This makes our TG able to capture the inherent non-stationarity present in the traffic generated by the processors. We validate the precision of our cycle-true traffic simulation in systemC. We show that the network latency, transaction delay and aggregated throughput of a complete SoC platform are very close when we use TGs replacing processors. With our environment, the designer can explore the design space in a very flexible manner by, for instance, exploring other network architectures on a single phase in which network contention occurs.

The paper is organized as follows. In Section 2 we review the existing techniques for onchip traffic generation. Section 3 presents our traffic generator and the flow that we propose for analyzing and synthesizing on-chip traffic. In Section 4 we describe the simulation environment we have used and finally, Section 5 presents experimental results focussed on our traffic generation environment accuracy. 


\section{On-chip traffic generation}

Using traffic generator in a simulation environment involves the following steps: $i$ ) the IP designer collects simulation traces by observing the behavior at the interface of each master component (if the IP is available), $i i$ ) he builds traffic models as close as possible to these traces, iii) the platform designer instantiates a traffic generator for each master component based on these models, and $i v$ ) inserts them in the simulation platform in place of the original components. Traffic generators can be separated into two main categories: the deterministic approach, in which traffic is produced using a finite state machine (FSM) configured by the IP designer or using a previous simulation trace, and the stochastic approach, in which the traffic is produced by a parameterized non-deterministic process. In this section, we shortly review these two methods with their pros and cons for on-chip traffic modelling.

\subsection{Deterministic traffic generation}

A deterministic traffic generator $[6,11,10]$ is derived from real simulation traces or written from scratch by IP designers. Such a TG can generate accurate transactions in time, size, and idle time that match the behavior of an IP. The advantages of such traffic generators are their precision and the speedup factor that they can achieve compared to the complete IP simulation.

For example, the TG proposed in [11] uses a trace compiler that can generate a program for a reduced instruction set processor that will replay the recorded transactions in a cycle-accurate simulation without having to simulate the complete processor. This TG is sensitive to the network latency: changing network latency will produce similar effect on the TG and on the original IP. This is a very important point which is also taken into account in our environment.

One limitation of the deterministic approach is that the length of the simulation is limited by the length of input traces used. Furthermore, such TGs cannot handle behaviors that are dependent on input data sets.

\subsection{Stochastic traffic generation}

An alternative solution is to use stochastic traffic generators. These TGs are useful either when the IP is not fully available or when the behavior is likely to change from one execution to the other. However some traffic are very difficult to model and the traffic generation environment should include advanced statistical analysis tools.

The major part of NoC performance evaluation is currently done using random sources [18, 16, $9,13]$. These works mainly focus on the evaluation of the NoC in its early stages of development, and on its performance under random traffic. For example, [9] uses stochastic models for generating transaction sizes and transaction delay using several statistical laws (Poisson, Exponential, and Normal). However none of these works propose a fitting procedure to determine the adequate statistical models that should be used to simulate the traffic. Moreover, all these works consider first order statistics only.

Second order statistics in on-chip traffic have been introduced by Marculescu in [17]. They have isolated a long-range-dependent behavior (LRD) in the amount of data transferred between different modules of an MPEG-2 decoder. Long-range-dependence is a very significant characteristic because it has been shown to have a strong impact on network performance [17, 12]. Not taking LRD into account may lead to a dramatic under-estimation of needed buffer sizes in the network. Our framework integrates second order statistics analysis and fitting procedures.

LRD behavior has been widely found on Internet traffic [5] and demonstrated for on-chip multimedia traffic at the coarse-grain level [17]. The important difference with the work presented in [17] is that our simulation occurs at a cycle-accurate level leading to important results concerning some problems mentioned above (cache behavior, network contention). 


\section{$2.3 \quad$ Traffic models}

A stochastic model considers the traffic as realizations of stochastic processes, of which we will consider the following two main characteristics :

- First order statistics represent how the values of a process are distributed. They are fully described by the cumulative distribution function ( $\mathrm{CDF}$ ) or its derivative, the probability distribution function (PDF). It corresponds basically to the frequency of apparition of each possible value taken by the process. This function can be either adjusted to some classical law (Normal, Exponential, ...) or kept as it is which we will further refer to as custom-PDF.

- Second order statistics represent how values of the process are correlated at all possible lag. The covariance function $\gamma(l)=\mathbb{E}(X(t) X(t+l))-\mathbb{E}(X(t)) \mathbb{E}(X(t+l))$ of a process $\{X(t)\}_{t \in \mathbb{N}}$ holds such an information, e.g. how random variables distant of $l$ samples influence each other $(\mathbb{E}$ is the expectation). Bursty behavior (the ability of having some very busy periods and some very low ones), is stochastically characterized by the covariance function. The shape (especially the decay velocity) of the covariance function characterized the bursty behavior of the traffic at each scale.

Processes are described by a couple (First order statistics / Second order statistics). All the considered processes are stationary processes, that is to say their statistical properties (mean and covariance function) do not change in time.

We present now the stochastic processes we use to model on-chip traffic in our traffic generation environment.

\subsubsection{First order statistics}

We will consider the following models characterizing the probability distribution function of a process :

- $\operatorname{Normal}\left(\mu, \sigma^{2}\right)$ : This is a classical law, characterized by its mean $(\mu)$ and variance $\left(\sigma^{2}\right)$. It is widely used because the central limit theorem stipulates any independent statistical law converges towards a Normal law when the number of observation increases.

- Exponential $(\mu)$ : The exponential law with parameter $\mu$ (the mean) is commonly used to characterize inter-arrival times.

- $\operatorname{Gamma}(\alpha, \beta)$ : This statistical law is interesting because it encompass behaviors ranging from the exponential law $(\alpha=1)$ to the normal law $(\alpha \rightarrow+\infty)$. $\beta$ is a scale parameters used for adjusting mean and variance to the data.

- CustomPDF : When the PDF of the data under analysis cannot be well adjusted to any available statistical law, we record the frequency of apparition of each possible value of the process.

\subsubsection{Second order statistics}

For second order statistics, we will consider two types of processes : the classical ones, which have a fast decaying covariance function and the long-range-dependent ones which on the contrary exhibit a slowing decaying covariance function.

We consider two classical models :

- IID (Independent Identically Distributed) processes have no correlations at all. Each random variable of the process is independent of the others. As a consequence, bursty behavior cannot be taken into account. This is the main limitation if IID process, and this is why they IID processes cannot be used as an accurate representation of traffic.

- ARma (Auto Regressive Moving Average) processes correspond to the filtering of IID processes, introducing some correlations between random variables. The covariance function, 
controlled by two polynomials $\Phi$ and $\Theta$, decays exponentially and is therefore only able to reproduce small-scales correlations. Bursty behavior can hence be taken into account at small scales but not at large scales. Higher order polynomials increases the scale of the correlation but also increases the number of parameters to estimate. This leads to a complex fitting procedure. For large scale bursty behavior, long-range-dependent processes have been introduced [17].

Long-range-dependence (LRD) [4] is a very significant statistical characteristic because it has been shown to have very important impact on network performance $[17,12]$. Not taking LRD into account leads to a dramatic under-estimation of needed buffer sizes in the network.

LRD processes are characterized by a slowly decaying covariance function that is no more summable. Data are correlated over a non-limited range of time lags and this property results in a scale invariance phenomenon. No characteristic time scale can be identified in the process, they are all equivalent for describing its statistics: the part resembles the whole and vice e versa. This is why LRD is also called Self-Similarity ${ }^{1}$. LRD implies in the scope of traffic analysis a bursty behavior over a range of time scales. Long-range-dependence is fully described by the Hurst parameter $H$ that controls the decaying velocity of the covariance function, and therefore how much the process is self-similar. $H$ varies from 0.5 , which corresponds to the IID case (no LRD), to 1 . If $H$ is near or above 1, LRD is not sufficient to model the behavior, it can be the signature of the non-stationarity of the trace.

The LRD processes that we consider are the following:

- FGN (Fractional Gaussian Noise). This process is a classical stationary LRD process. Its covariance function exhibits a power-law decay, whose exponent is directly related to the Hurst parameter. Originally it is a Gaussian process, the generation of non-Gaussian version of this process is discussed in Section 3.4.

- FARIMA (Fractionally Integrated ARMA) processes combine both the short-range correlations of an ARMA process and the long-range ones introduced by the fractional integration. Both behaviors have distinct parameters, basically an LRD parameter $H$ is added to the ARMA parameters. This is a versatile model adaptable to many situations.

\subsection{Phases in traffic}

A processor associated with a cache generates a non-stationary traffic, which can be divided into phases corresponding to different parts of the executed program. Some phases are stationary, some other are very hard to model statistically because they are non-stationary. A complete traffic generation environment should integrate both deterministic and stochastic traffic generation techniques. We refer to this as multi-phase traffic generation.

In this report, results only consider deterministic replay and IID stochastic processes. The complete study of ARMA and long-range-dependant modelling is part of on-going work.

\section{Multi-Phase Traffic Generator}

We now present our analysis and synthesis flow for building multi-phase traffic generators that can be used to replace an IP in cycle-accurate NoC performance evaluation.

\subsection{On-chip traffic modeling}

The traffic produced by a component is modeled as a sequence of transactions. The $k^{t h}$ transaction is a 4-uple $(A(k), C(k), S(k), D(k))$ meaning in this order, target address, command (read or write), size of transaction, and delay (number of cycles between two successive requests). This is illustrated in Figure 1. From this transaction sequence, we define the aggregated throughput $W_{\Delta}(k)$,

\footnotetext{
${ }^{1}$ Actually, LRD strictly corresponds to asymptotic second order self-similarity.
} 
which corresponds to the amount of bus-words transferred in the time interval $[k \Delta,(k+1) \Delta]$. We also define the latency of the $k^{t h}$ transaction $L(k)$ as the number of cycles between the start of a $k^{\text {th }}$ request and the start of the associated response. This is basically the round-trip time in the network.

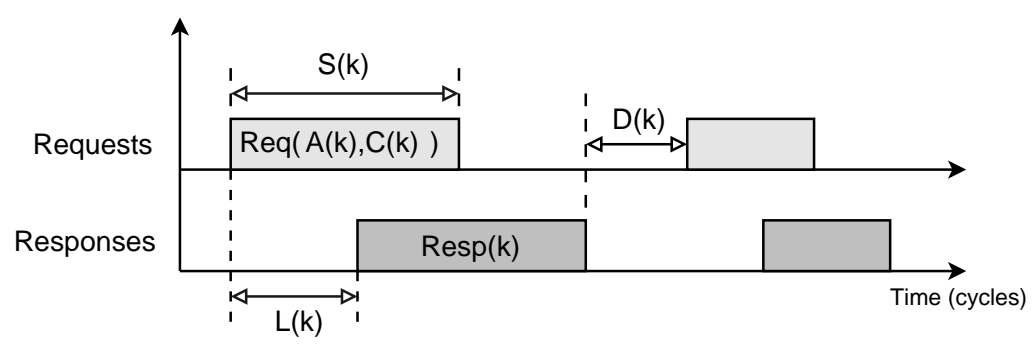

Figure 1: Traffic modelling formalism

Traffic is generated according to the 4-uple describing each transaction, and this 4-uple can be either read from a previously recorded trace (replay), or generated as the realization of a stochastic process. In the later case, we will consider that addresses and commands are not independent from each other, but all other correlation will not be taken into account. For now, delay and size of a transactions will be considered independent.

\subsection{Global methodology}

The global simulation flow is depicted on Figure 2. First, we generate a reference trace by simulating the processor IP to be emulated. The trace is itself extracted from a vCD (Value Change Dump) file that record selected signal variations during the simulation. For our purpose, we need to record some signals of the VCI (Virtual Component Interface) interface of the IP we want to replace with a traffic generator. This trace is obtained with an ideal network environment (no network contention), which makes the simulation very fast. Then, we process the trace in our traffic analysis and synthesis tool explained hereafter and we obtain configuration files for our traffic generators. A generic traffic generator has been written once for all, it is referred to further in the text as MPTG. Transactions are generated by MPTG according to a phase description file and a sequencer is in charge of switching between phases. Each phase consists either of a replay of a recorded trace, or of a stochastic model with parameters adjusted by the fitting procedure described in next section. Finally, the platform designer describes the desired platform architecture (such as the ones presented in Figure 5) and uses a perl script (referred to as SocGen) that generates all files needed for simulation. The simulation takes place and performance analysis indicates whether some parameters of the platform have to be changed or not.

\subsection{Traffic analysis}

The designer can choose to analyze the traces based upon any of the series introduced in Section 3.1. In particular, the traffic can either be generated using the delay and size of transaction, or the aggregated throughput which combines both. In the later case, a constant delay is computed for each time window.

- Segmentation: The first step of the analysis is the segmentation, it consists in identifying phases in the trace with a regular behavior. Currently the segmentation is done manually. This resulted in four phases in MP3 and four phases in MPEG. We are currently working on automating this part, which is a classical problem in trace analysis, but our results are too preliminary to be presented here. Intuitively, the segmentation should result in 2 phases per frame on the MP3 application.

- Compression: For deterministic traffic generation, each transaction 4-uple is recorded in a file and compressed using the BZ2 program, which results on average in dividing the size of the trace 


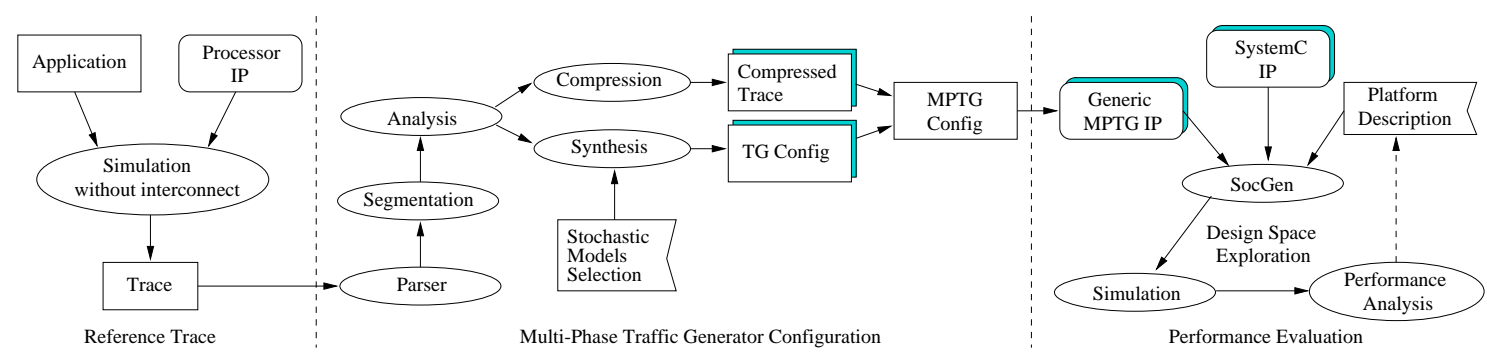

Figure 2: MPTG Framework: Traffic analysis and synthesis flow

by 70 . The resulting trace can be directly replayed by our MPTG.

- Statistical analysis: Statistical analysis is performed on each extracted phase by an automatic fitting procedure that adjusts the first and second statistical orders (see [15] for details). The designer has to choose which model he wants to use (with or without LRD for instance) before analyzing the trace. The probability distribution function (PDF) (first statistical order) can be either fitted to some classical distributions (Gaussian, Exponential, Gamma, Log-Normal, ...) or kept as it is (the model is then the probability of apparition of each value of the process). The fit is done using Maximum Likelihood Expectation and a $\chi^{2}$ goodness-of-fit test is used to compare and evaluate all different solutions [8]. The covariance function (second statistical order) can be fitted to an ARMA (short range correlations only), Fractional Gaussian noise (long-range-dependence only), or a FARIMA (both short and long-range correlations). We use a wavelet-based estimation of the Hurst parameter [3] widely adopted in the network traffic analysis domain (see [2]).

\subsection{Traffic synthesis}

Traffic analysis returns a collection of model instances associated to each identified phase of a time series, that can be used to generate synthetic traffic.

We developed an independent random number generator that can produce realizations of a wide variety of processes including non-gaussian long-range-dependant ones [15]. This generator is integrated in the MPTG and the analysis simply produces the adequate MPTG configuration file.

\subsection{Traffic generator key features}

As a summary, we highlight the main characteristics of our MPTG:

- It takes into account network properties (latency, contention, etc.). This means that the MPTG will offer a good emulation of the processor independently of the network it is connected to. Hence, we can re-use the same configuration file for many different platforms.

- If, for some reason, the designer is not satisfied with the analysis based on aggregated throughput, other characteristics presented in section 3.1 can be used.

- The global methodology presents as few as possible manual editing (e.g. the complete simulation platform is generated). Moreover, the traffic analysis and synthesis flow can be very easily adapted to another synthesis environment; only the MPTG IP has to be re-written (assuming that trace parsing is a necessary tool in any performance analysis environment).

- Our MPTG is able to run processes having a wide variety of parameters: deterministic replay, various stochastic models with prescribed first and second order statistics.

- Our MPTG is multi-phase and hence can emulate a wide variety of behavior alternating, for instance, replay and stochastic traffic generation. NoC.

With all these features, the designer has a very flexible tool for the design space exploration of 


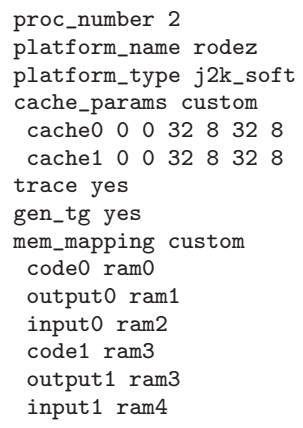

interconnect dspin

tg0 02

mips0 12

ramo 20

ram1 10

ram2 $2 \begin{array}{lll}2 & 1\end{array}$

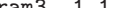

ram4 2 2 2

tty 00

req_fifo_size 8

rsp_fifo_size 8

Figure 3: Platform description from which the platform of Fig. 5 (left) it is generated

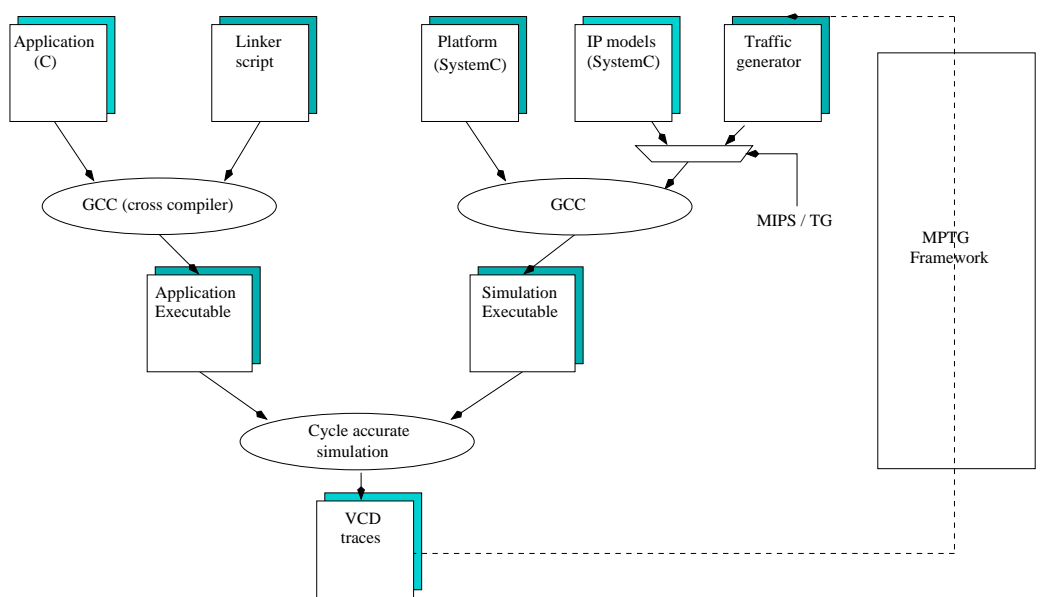

Figure 4: Complete simulation flow with MPTG framework

\section{Simulation environment and set-up}

\subsection{SocLib}

The results presented in this paper have been obtained in the SoCLib simulation environment [1]. SoCLib is a library of open-source cycle-accurate SystemC simulation models. Example of simulation models available in SoCLib are: a MIPS R3000 processor (with its associated data and instruction cache), standard on-chip memories, DMA controller and several kinds of networkson-chip. Two NoC simulation models are available in SoCLib: Spin [7] and DSpin which is a distributed version of Spin with many improvements, both have been provided by the Lip6 laboratory. A typical SoCLib platform is represented in Fig. 5 (left). It is composed of two master components (a MIPS and a TG) and five memories. The network-on-chip we use is a set of 4 ports routers that can be interconnected in mesh topology in order to provide the desired packet switched network architecture.

\subsection{Simulation flow}

The software running on the processors are compiled with the GNU gcc tool suite. We used a tiny operating system called mutek [14]. The software compilation flow is depicted on the left of Fig 4.

Interconnecting the different SystemC models to obtain a working SoC platform is not an easy task because each VCI (Virtual Component Interface, used in SoCLib models) port is composed of 160 wires. A script (called SocGen) has been written to generate automatically the SystemC interconnection file from a very simple text file describing the components used in the platform and the architecture of the network (Fig. 3). This script also generates software configuration 
files (Makefile, linker script) and the simulation can immediately take place. Fig. 5 (left) shows a particular platform generated by SocGen from the specification shown on Fig. 3. Note that the designer explicitly specifies the architecture of the network, i.e which processor is connected to which router. We think that a platform generator tool like SocGen is an essential component of any SoC simulation environment.

\section{$5 \quad$ Experimental results}

In this section, after discussing the simulation time issue, we present experimental results showing that the multi-phase feature is mandatory to provide realistic traffic.

\subsection{Applications}

Here follows a description of each application code we have used.

- JPEG: The JPEG image decompression standard. We use two implementation, the first one is the libjpeg library (http://www.ijg.org/), further referred to as JPEG and the second one is a in-house multi-thread implementation (further referred to as MJPEG). Both implementations decode a 64x64 pixels image.

- JPEG2000: Standard implementation of the j2k image compression standard (http://j2000.almacom.com/). It decodes a 64x64 image.

- MP3: Audio decompression standard (http://www. underbit.com/products/mad/). It decodes two MP3 frames of an audio file.

- MPEG-2: Video decompression standard (http://libmpeg2.sourceforge.net/). It decodes two images (a P and then a I).

Each application code needed to be adapted to run on the platforms. This typically implied fixed precision conversion (the processor in the SoCLib environment is a MIPS R3000 without floating point unit), replace I/O with basic read/write operation in memory as no file system is included in mutek, and writing linker scripts in order to specify the memory organization and mapping.

\subsection{Platforms}

We performed experiments with the four application introduced in the previous section (MP3, JPEG2000, both JPEG implementations, and MPEG).

We have used two SoC platforms:

- The DIRECT platform does not use any interconnect, the processor is directly connected to a memory holding all necessary data. The latency is thus constantly equal to 1 cycle. This platform is used for basic validation of the MPTG and for reference trace collection as shown in Figure 2.

- The MESH platform is shown in Figure 5. The components are interconnected with the DSPIN NoC. The MIPS processor of Figure 5 is running the application. The BACK TG of Figure 5 is used for introducing contention over the network. It sends requests to both memories RAM TG1 and RAM TG2, whereas the MIPS communicates with the three other memories used for code, data, input and output streams. In order to test the MPTG in a more realistic way, we use two BACK TG. In the first one (this platform is later referred to as MESH1), the BACK TG produces a random traffic (exponential sizes with mean 15 words and exponential inter-arrivals with mean 35 cycles), and the second one (referred to as MESH2) alternates between two phases, one with a high communication load (exponential 


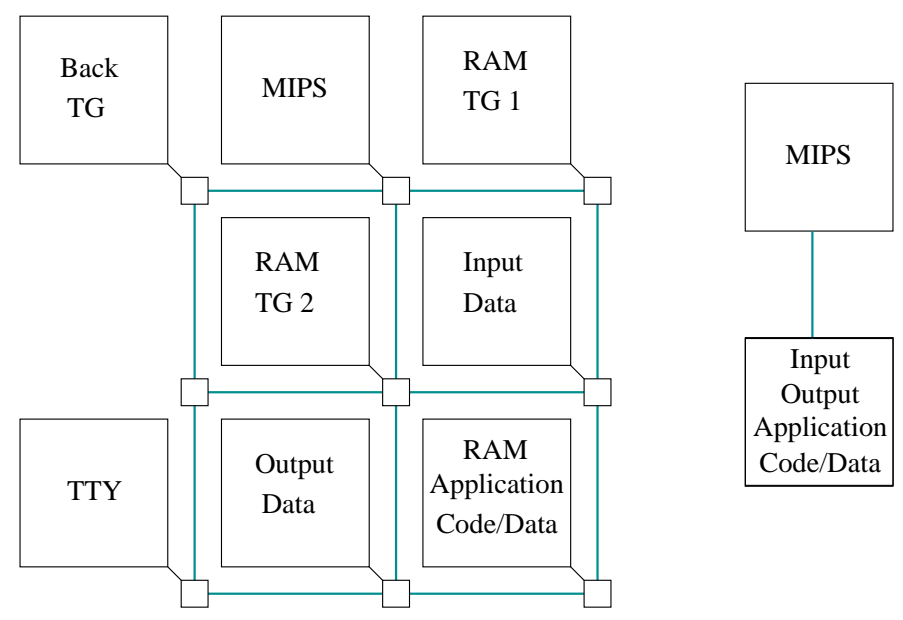

Figure 5: Simulation platforms: DIRECT (right) and MESH (left)

sizes with mean 20 words and exponential inter-arrivals with mean 5 cycles) and another with a low one (exponential sizes with mean 10 words and exponential inter-arrivals with mean 50 cycles). This introduce a time-varying contention and approximately multiply the number of cycles of the execution by 4 .

\subsection{Design time setup}

We explain here the work needed to setup a NoC performance evaluation platform and give an estimation of the additional design effort needed to use MPTG in the design flow. The different steps of the methodology are the following:

- Generation of the initial platform. It consists in writing the configuration files presented on Fig 3 and running the SocGen script to generate the main SystemC file which is immediate.

- Initial simulation of a MIPS platform. Simulation times are analyzed in the next section.

- Trace analysis and phase determination. Trace analysis takes approximately a minute for a 5 million cycles simulation. Phase determination is currently done manually. The trace given in Fig. 6 represents two frames decoded by the MP3 application. The MP3 application is decomposed in a repetitive sequence of two phases, this can be easily detected as seen on Fig 6. Our goal is to propose a framework in which the designer will choose a number a phases (which indicates the precision of the decomposition), and the system will determine the phases automatically.

- TG configuration file generation is immediate once the designer has chosen the phases

- Simulation with MPTG. Simulation time are analyzed in next section.

Except for the phase identification process, we can safely affirm that no additional time is spent by the designer when using MPTG, provided he is used to MPTG tools. Most of the time will be spent in initial and final simulation. An important feature of our MPTG framework is that when changing a parameter of the platform (e.g. the architecture or the router's buffer size) only the MPTG platform has to be simulated again.

\subsection{Simulation time}

We compared the simulation time of the MP3 decoder application running on the original MIPS and on the corresponding generated TG. When the number of processor is more than one, each processor executes the same code (with different data and input memory spaces). Simulation results are shown in table 1, the rows labelled MIPS Sim. Time corresponds to the simulation of a platform including MIPS processors (with data and instruction cache) and memories. The Row labelled MPTG Sim. Time corresponds to simulation in which the TGs replaces the MIPS and cache.

The simulation time can be split in several parts: 


\begin{tabular}{|c|c|c|c|c|}
\hline Number of processors & 1 & 2 & 3 & 4 \\
\hline mesh size & $0 \times 0$ & $2 \times 2$ & $3 \times 3$ & $4 \times 4$ \\
\hline MIPS Sim. Time & 35.44 & 142.5 & 304.5 & 683.50 \\
\hline MPTG Sim. Time & 15.05 & 102.9 & 190.9 & 406.3 \\
\hline Speedup & 2.35 & 1.38 & 1.59 & 1.68 \\
\hline
\end{tabular}

Table 1: Simulation time (s) of MIPS versus various generated TG $\left(5.10^{6}\right.$ simulated cycles for the MP3 application)

- SystemC core: this is the SystemC discrete event simulator engine. This time cannot be diminished.

- Component models: this is the time spent in the computation of the state machine associated with each component. The time spent in each component depends on the degree of details of the modeling and the activity of the component. The interconnection system holds much of the simulation time because its activity is very high (it is activated every cycle). The MIPS r3000 processor model works basically like an ISS (Instruction Set Simulator). A two-stages pipeline is emulated, but the precise hardware architecture is not modelled. Therefore the MIPS is not using a major part of the simulation time and this explains why the speed-up factor is not very high (see below). We would expect an higher speedup with a more complex IP, however we do not expect a dramatic increase of this speedup because still the interconnection system holds the major part of it as its activity is related to the sum of all connected component activity.

- VCD output: this is the time spent for writing value changes of specified signals. This accounts for a non-negligeable part of the simulation time, as we noticed the simulation runs twice faster without VCD dump than with VCI interface signal dump (dumping the clock makes it again two times slower). Note that in our traffic generation environment, this VCD output needs to be collected only once, on a reference platform that can be without interconnect (see Section 3.2), traffic generators are derived from this reference trace, and further simulations for performance evaluation can be run without VCD tracing.

We insist on an important point here, the measures presented in this section do not exhibit a dramatic reduction in simulation time (between 1.5 and 2.5) because much of the simulation time is spent in the interconnection system. We argue that the real benefit of a traffic generation environment lies in the flexibility of the tool. For instance, the application probably have a particular phase during which most of the NoC problems occur. Simulating only this phase is very easy with MPTG just by keeping some phases in the TG configuration file, while this is often much more difficult to isolate subsets of program execution from the whole execution of the application. Another important point is that TGs can be used without having access to the source code of the original IPs, which is useful if network prototyping is done by a third party.

\subsection{Manual phase identification}

For each application code, we first executed the software on a MIPS (with its associated instruction and data cache) directly connected to a memory, and recorded the communication trace at the VCI interface of the cache. This trace was manually segmented into phases. Table 5.5 show the number of phases found for each application and this is illustrated on Fig. 6 for the MP3. One can see on Fig. 6 that P0 and P1 appear twice (because 2 frames are decoded), so the phase number is 2 , and the total phase number is 4 in table 5.5. The segmentation was done on the aggregated throughput time series. 


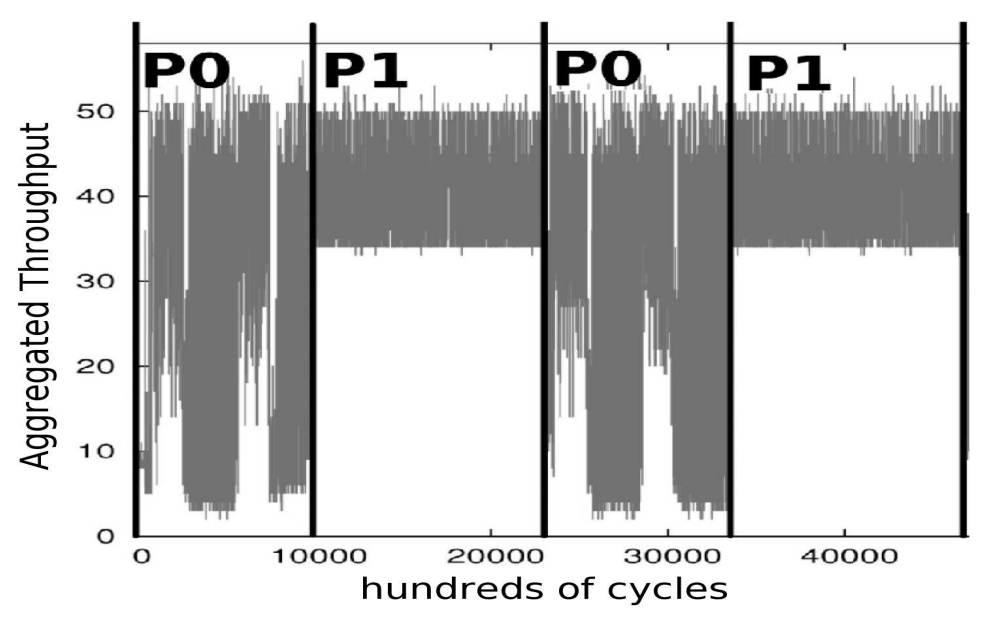

Figure 6: Segmentation of the MP3 aggregated throughput into two phases

\begin{tabular}{|c|c|}
\hline Application & Phase Number (Total) \\
\hline MP3 & $2(4)$ \\
\hline JPEG & $1(1)$ \\
\hline JPEG2000 & $3(3)$ \\
\hline MPEG & $2(4)$ \\
\hline
\end{tabular}

Table 2: Phase number from the manual segmentation of traces. The phase number is the number of distinct identified phases, and total number is the number of phases in the complete trace.

\subsection{MPTG accuracy}

We then followed the flow of Fig. 4 and generated four different traffic generators:

- DR: it corresponds to a replay of the recorded trace of transactions of the reference simulation.

- TPR: it corresponds to a replay of the aggregated throughput (size and target address are recorded also).

- MPTG: the TGs take the identified phases into account and the probability distribution function is taken into account per phase. Only IID processes are considered. The study of ARMA and long-range dependant processes is on-going work.

- STG: the TGs generate a mono-phase stochastic traffic with throughput mean and variance adjusted to the reference trace

Simulations have been performed for each traffic generator configuration/ platform pair, and we hereafter present the collected results.

In order to compare a given configuration with the reference MIPS one, we should not look at global metrics such as the average delay or the average throughput. This would not point out the interest of the multi-phase approach. So, we have defined an accuracy measure that can be computed on each metric (delay, size, command and throughput). We compare the mean evolution of the metrics. We define the error between two simulations for a given metric $M$ as the mean of absolute values of relative differences between two mean evolutions. Let $M_{r e f}(i)$ be the mean evolution of some metric (computed in non-overlapping blocks of size $\delta$ ) for the reference simulation, let further $M(i)$ be the mean evolution of the same metric for another simulation, and let finally $n$ be the number of points of both functions. The error (in percent) reads:

$$
E_{\delta}=\frac{1}{n} \sum_{i} \frac{\left|M_{r e f}(i)-M(i)\right|}{M_{r e f}(i)} * 100
$$


This can be done in a multi-resolution way by using several block size $\delta$ on which the mean evolution will be computed. This error is reported in tables 3, 4, 5, 6, and 7 for respectively the MP3, MJPEG, JPEG, j2k, and MPEG applications. In these tables, the block size $\delta$ is fixed to 5000 samples. We have checked that the error is stable with $\delta$.

The mean evolution of the aggregated throughput is depicted on Fig. 7 and 8, for the MP3 application and for various block sizes. The oscillation in the Fig. 8 is due to the BACK TG that alternates between two phases so that the contention on the network is periodically varying in time.

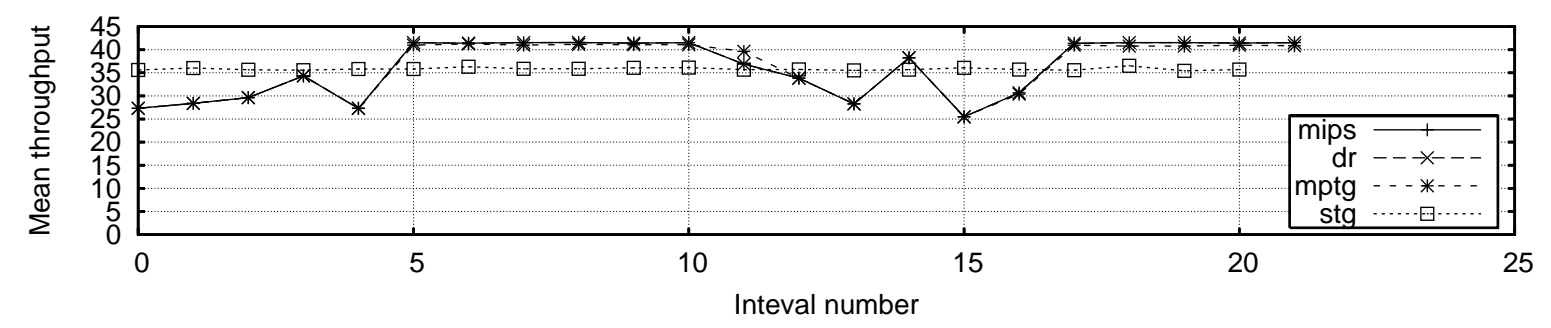

MP3 - Block size 10000

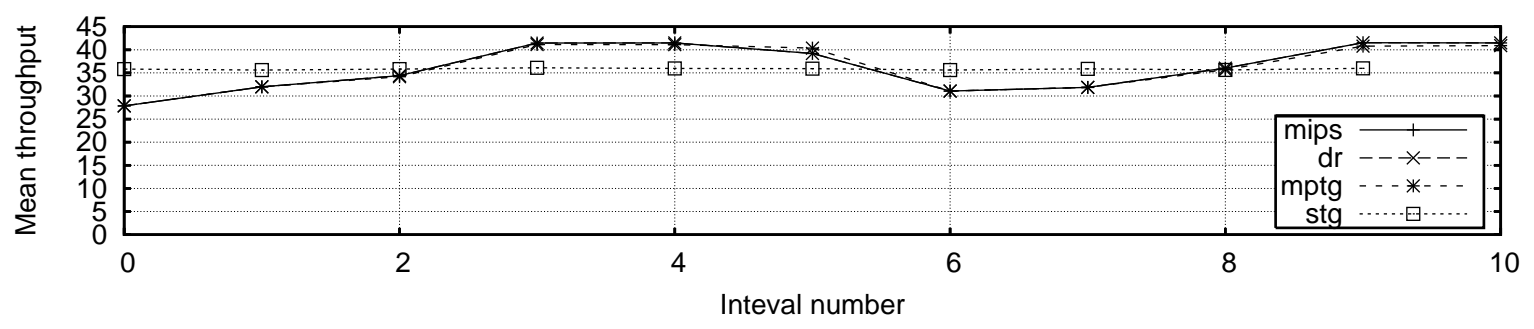

MP3 - Block size 20000

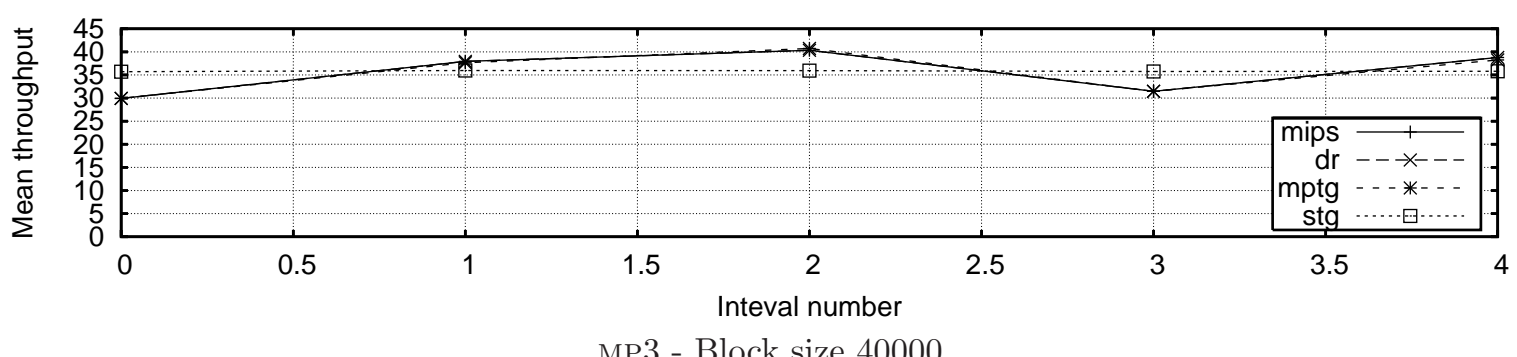

Figure 7: Mean evolution of the aggregated throughput, for the MP3 application on the DIRECT platform, for various block size. 

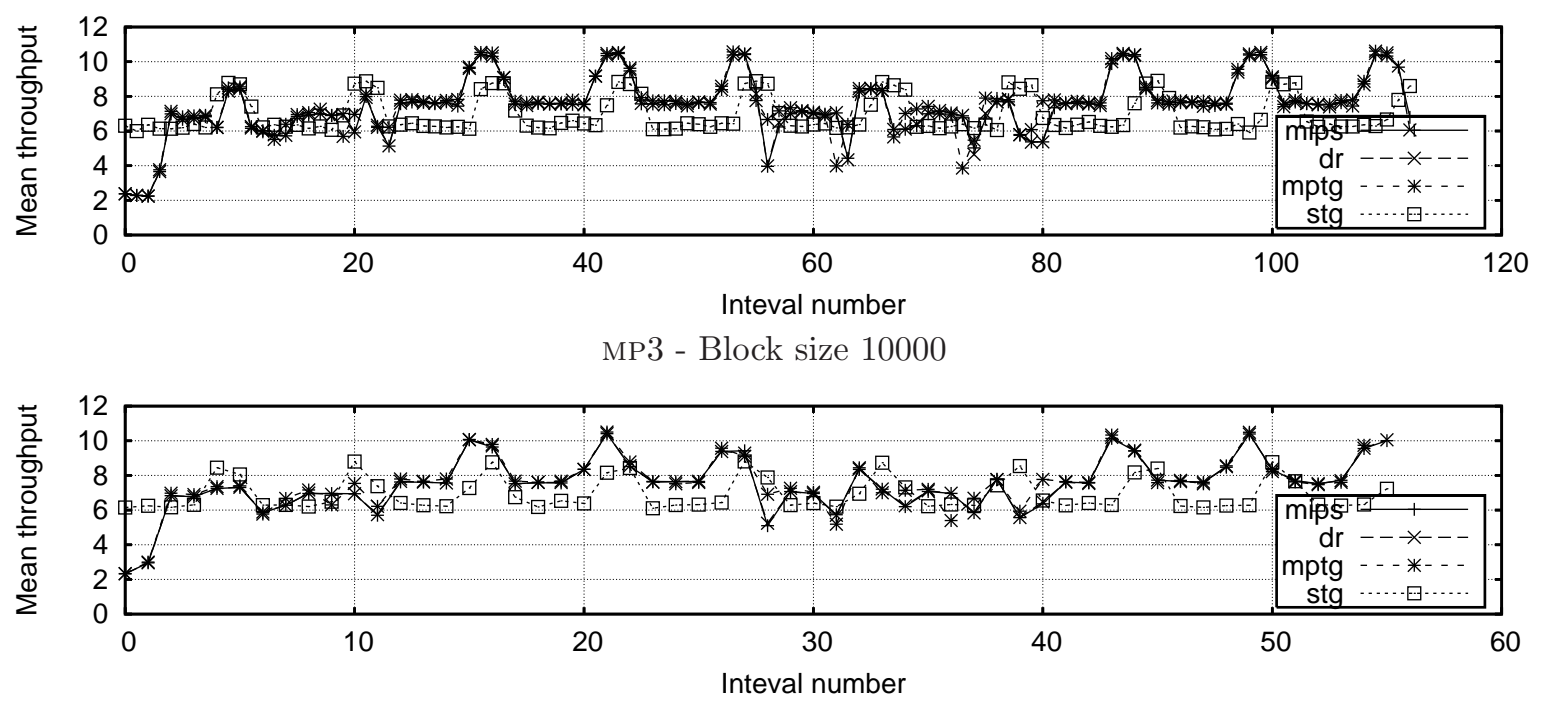

MP3 - Block size 20000

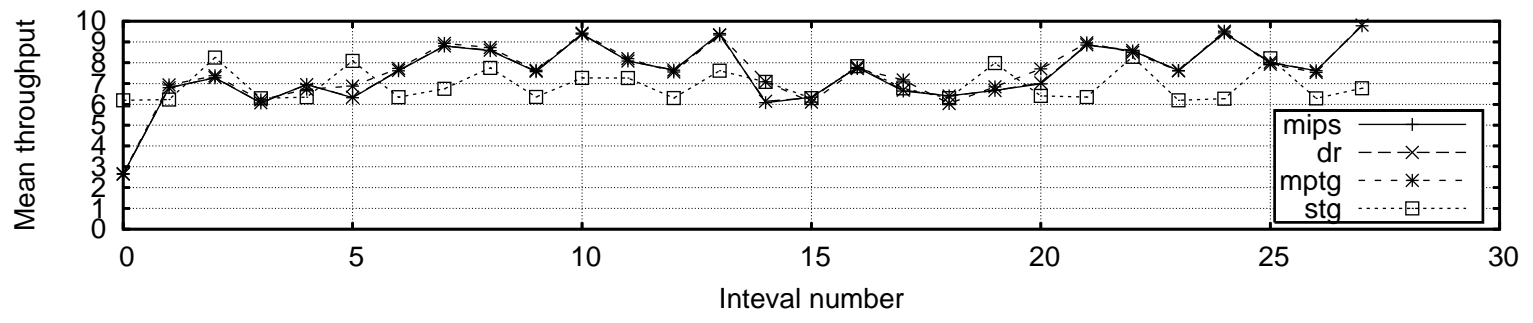

MP3 - Block size 40000

Figure 8: Mean evolution of the aggregated throughput, for the MP3 application on the MESH2 platform, for various block size.

\begin{tabular}{|c|c|c|c|c|c|c|}
\hline Platform & Config & IA & Size & Cmd & Agg & Lat \\
\hline \hline direct & $\mathrm{dr}$ & 0 & 0 & 0 & 0.002 & 0 \\
\hline direct & $\mathrm{tpr}$ & 0.002 & 0 & 0 & 0 & 0 \\
\hline direct & $\mathrm{mptg}$ & 0.183 & 1.759 & 0.883 & 0.487 & 0 \\
\hline direct & $\mathrm{tg}$ & 6.293 & 13.444 & 6.359 & 2.898 & 0 \\
\hline \hline rodez1 & $\mathrm{dr}$ & 1.152 & 0 & 0 & 0.127 & 0.047 \\
\hline rodez1 & $\mathrm{tpr}$ & 21.649 & 0 & 0 & 2.472 & 0.194 \\
\hline rodez1 & $\mathrm{mptg}$ & 18.976 & 1.656 & 0.826 & 1.875 & 0.342 \\
\hline rodez1 & $\mathrm{tg}$ & 20.244 & 13.988 & 6.335 & 12.129 & 0.532 \\
\hline \hline rodez2 & $\mathrm{dr}$ & 1.139 & 0 & 0 & 0.209 & 0.088 \\
\hline rodez2 & $\mathrm{tpr}$ & 21.654 & 0 & 0 & 3.249 & 1.139 \\
\hline rodez2 & $\mathrm{mptg}$ & 19.138 & 1.614 & 0.801 & 2.121 & 1.257 \\
\hline rodez2 & $\mathrm{tg}$ & 20.560 & 13.790 & 6.369 & 14.818 & 0.630 \\
\hline
\end{tabular}

Table 3: Error (in percent) on various metrics with respect to the reference Mips simulation for the MP3 application on various platforms. 


\begin{tabular}{|c|c|c|c|c|c|c|}
\hline Platform & Config & IA & Size & Cmd & Agg & Lat \\
\hline direct & $\mathrm{dr}$ & 0 & 0 & 0 & 0.001 & 0 \\
\hline direct & $\mathrm{tpr}$ & 0.002 & 0 & 0 & 0 & 0 \\
\hline direct & $\mathrm{mptg}$ & 1.058 & 0.609 & 0.238 & 0.390 & 0 \\
\hline direct & $\mathrm{tg}$ & 5.557 & 6.351 & 0.345 & 1.065 & 0 \\
\hline \hline rodez1 & $\mathrm{dr}$ & 1.127 & 0.000 & 0.000 & 0.361 & 0.076 \\
\hline rodez1 & $\mathrm{tpr}$ & 26.733 & 0.000 & 0.000 & 2.472 & 0.313 \\
\hline rodez1 & $\mathrm{mptg}$ & 23.333 & 0.571 & 0.224 & 1.965 & 0.349 \\
\hline rodez1 & $\mathrm{tg}$ & 33.361 & 6.308 & 0.325 & 6.164 & 0.564 \\
\hline \hline rodez2 & $\mathrm{dr}$ & 1.127 & 0.000 & 0.000 & 0.326 & 0.231 \\
\hline rodez2 & $\mathrm{tpr}$ & 26.733 & 0.000 & 0.000 & 2.534 & 1.725 \\
\hline rodez2 & $\mathrm{mptg}$ & 23.317 & 0.624 & 0.245 & 2.101 & 1.683 \\
\hline rodez2 & $\mathrm{tg}$ & 33.125 & 6.355 & 0.307 & 7.957 & 1.916 \\
\hline
\end{tabular}

Table 4: Error (in percent) on various metrics with respect to the reference Mips simulation for the MJPEG application on various platforms.

\begin{tabular}{|c|c|c|c|c|c|c|}
\hline Platform & Config & IA & Size & Cmd & Agg & Lat \\
\hline direct & $\mathrm{dr}$ & 0 & 0 & 0 & 0.001 & 0 \\
\hline direct & $\mathrm{tpr}$ & 0.002 & 0 & 0 & 0 & 0 \\
\hline direct & $\mathrm{mptg}$ & 3.841 & 1.696 & 0.426 & 1.522 & 0 \\
\hline direct & $\operatorname{tg}$ & 6.002 & 6.521 & 0.470 & 0.990 & 0 \\
\hline \hline rodez1 & $\mathrm{dr}$ & 0.243 & 0 & 0 & 0.251 & 0.059 \\
\hline rodez1 & $\mathrm{tpr}$ & 28.627 & 0 & 0 & 3.190 & 0.285 \\
\hline rodez1 & $\mathrm{mptg}$ & 29.605 & 1.687 & 0.423 & 3.620 & 0.351 \\
\hline rodez1 & $\operatorname{tg}$ & 40.793 & 6.503 & 0.471 & 5.367 & 0.435 \\
\hline \hline rodez2 & $\mathrm{dr}$ & 0.243 & 0 & 0 & 0.228 & 0.095 \\
\hline rodez2 & $\mathrm{tpr}$ & 28.627 & 0 & 0 & 3.552 & 1.407 \\
\hline rodez2 & $\mathrm{mptg}$ & 29.395 & 1.682 & 0.430 & 3.631 & 1.477 \\
\hline rodez2 & $\mathrm{tg}$ & 40.781 & 6.500 & 0.485 & 7.296 & 1.347 \\
\hline
\end{tabular}

Table 5: Error (in percent) on various metrics with respect to the reference Mips simulation for the JPEG application on various platforms. 


\begin{tabular}{|c|c|c|c|c|c|c|}
\hline Platform & Config & IA & Size & Cmd & Agg & Lat \\
\hline direct & $\mathrm{dr}$ & 0 & 0 & 0 & 0.001 & 0 \\
\hline direct & $\mathrm{tpr}$ & 0.002 & 0 & 0 & 0 & 0 \\
\hline direct & $\mathrm{mptg}$ & 8.127 & 4.506 & 1.617 & 4.726 & 0 \\
\hline direct & $\mathrm{tg}$ & 24.465 & 20.553 & 6.161 & 16.890 & 0 \\
\hline \hline rodez1 & $\mathrm{dr}$ & 0.205 & 0 & 0 & 0.285 & 0.066 \\
\hline rodez1 & $\mathrm{tpr}$ & 15.719 & 0 & 0 & 11.629 & 0.205 \\
\hline rodez1 & $\mathrm{mptg}$ & 19.597 & 5.305 & 1.843 & 12.825 & 0.416 \\
\hline rodez1 & $\mathrm{tg}$ & 37.381 & 20.609 & 6.133 & 20.396 & 0.475 \\
\hline \hline rodez2 & $\mathrm{dr}$ & 0.205 & 0 & 0 & 0.553 & 0.222 \\
\hline rodez2 & $\mathrm{tpr}$ & 15.732 & 0 & 0 & 11.664 & 1.933 \\
\hline rodez2 & $\mathrm{mptg}$ & 19.597 & 5.382 & 1.857 & 12.464 & 1.649 \\
\hline rodez2 & $\mathrm{tg}$ & 37.413 & 20.462 & 6.153 & 21.388 & 1.720 \\
\hline
\end{tabular}

Table 6: Error (in percent) on various metrics with respect to the reference Mips simulation for the JPEG2000 application on various platforms.

\begin{tabular}{|c|c|c|c|c|c|c|}
\hline Platform & Config & IA & Size & Cmd & Agg & Lat \\
\hline \hline direct & $\mathrm{dr}$ & 0 & 0 & 0 & 0.001 & 0 \\
\hline direct & $\mathrm{tpr}$ & 0.004 & 0 & 0 & 0 & 0 \\
\hline direct & $\mathrm{mptg}$ & 2.781 & 1.509 & 0.371 & 0.698 & 0 \\
\hline direct & $\mathrm{tg}$ & 70.844 & 44.444 & 6.445 & 1.786 & 0 \\
\hline \hline rodez1 & $\mathrm{dr}$ & 9.067 & 0 & 0 & 1.480 & 0.073 \\
\hline rodez1 & $\mathrm{tpr}$ & 23.124 & 0 & 0 & 3.516 & 0.186 \\
\hline rodez1 & $\mathrm{mptg}$ & 13.195 & 1.397 & 0.360 & 2.351 & 0.402 \\
\hline rodez1 & $\mathrm{tg}$ & 60.986 & 44.352 & 6.547 & 36.893 & 1.706 \\
\hline \hline rodez2 & $\mathrm{dr}$ & 8.827 & 0 & 0 & 1.056 & 0.380 \\
\hline rodez2 & $\mathrm{tpr}$ & 22.963 & 0 & 0 & 2.956 & 1.015 \\
\hline rodez2 & $\mathrm{mptg}$ & 12.946 & 1.358 & 0.346 & 2.264 & 0.492 \\
\hline rodez2 & $\mathrm{tg}$ & 60.546 & 44.309 & 6.493 & 41.502 & 2.131 \\
\hline \hline
\end{tabular}

Table 7: Error (in percent) on various metrics with respect to the reference Mips simulation for the MPEG-2 application on various platforms.

\section{Deterministic replay (DR):}

The accuracy of replay is very good (no errors on the DIRECT platform, and a very low one on the MESH platform. This validates the fact that it is meaningful to record a trace on a platform without interconnection, and to replay it on another platform. This is because our traffic generator is aware of network contention: for read operations, the time spent waiting for the network to be ready do not cover the inter-transaction time, whereas for write operation, because the cache is write through and has got a write buffer, this waiting time is covered by the inter-transaction one. Our traffic generator has been therefore configured with blocking reads and non-blocking writes, because the IP it emulate (a MIPS r3000) is so. Note that our traffic generator is flexible and can emulates other communication schemes such as split transactions for dataflow IPs.

\section{Aggregated throughput replay (TPR)}

The accuracy of throughput-based replay is very good on all metrics except the delay, because a constant delay is computed in the aggregation window. If the designer is not much concerned 
about emulating the precise timing of each transaction, but want that the amount of traffic injected in the network to be as the real application, then this configuration is what he needs. The trace recorded is much smaller because the delay information is discarded.

\section{Multi-phase traffic generation (MPTG)}

The MPTG configuration is throughput-base, that is to say the aggregated throughput has been fitted in order to generate the traffic generator configuration. This statistical fit was done on each extracted phases independantly. This explains why the delay accuracy is low just as for the TPR configuration. However on all other metric the accuracy is reasonably good.

\section{Simple traffic generation (STG)}

The STG configuration error is always higher than for all other configuration, highlighting the fact the phase behavior needs to be taken into account.

\section{Discussion}

These results show that multi-phase stochastic traffic generation is worth a try for NoC prototyping. Even though it is not as precise as deterministic replay, the phase behavior of the IP is preserved, which is in our opinion a key point of emulating the true contention on the network. The choice between stochastic and deterministic traffic generation depends on the purpose of the study. For instance simple traffic generator such as the STG configuration is a good way to evaluate and compare routing strategies and other large scale design choices, whereas deterministic trace replay can provide very good accuracy for tuning the implementation details in the routers. We believe that the multi-phase stochastic traffic generation is interesting as a compromise between random and deterministic approaches. It combines a good accuracy and overcomes deterministic limitations (reference trace length). It especially provides the designer a phase description of traffic, and a stochastic model for each identified phase. This allows more flexibility in the traffic generation. For instance, the parameters of the models can be slightly changed in order to evaluate the robustness of the NoC.

\section{Conclusion}

In this report we presented our methodology for networks-on-chip performance evaluation. We explained how the MPTG was built and we showed that its ability to replay a trace obtained from a fast simulation can be used by a designer who wishes to prototype different network architectures.

The contribution of this paper is to show that MPTG is a very good candidate for NoC prototyping. We pointed out that multiple phases are necessary to emulate correctly a processor-like IP. We also show experimental results that validate the accuracy of the traffic generated by MPTG. This should convince the reader that multi-phase traffic generation is needed and that our environment provides good accuracy in traffic modelling.

Our current work is now to provide further experiments in which more network characteristics are analyzed with our MPTG. We are also actively investigating the automation of the trace segmentation. 


\section{References}

[1] Soclib simulation environment. On-line, available at http://soclib.lip6.fr/, 2005.

[2] P. Abry, P. Flandrin, M.S. Taqqu, and D. Veitch. Wavelets for the analysis, estimation and synthesis of scaling data. In K. Park and W. Willinger, editors, Self-Similar Network Traffic and Performance Evaluation. WILEY, 2000.

[3] P. Abry and D. Veitch. Wavelet analysis of long-range dependent traffic. IEEE Trans. on Info. Theory, 44(1):2-15, January 1998.

[4] P. Doukhan, G. Openheim, and M. Taqqu. Theory and Applications of Long Range Dependance. Birkhäuser, 2002.

[5] A. Feldmann, A. Gilbert, W. Willinger, and T. Kurtz. The changing nature of network traffic: Scaling phenomena. ACM/SIGCOMM Computer Communication Review, 2(28), April 1998.

[6] N. Genko, D. Atienza, G. De Micheli, J. M. Mendias, R. Hermida, and F. Catthoor. A complete network-on-chip emulation framework. In DATE 05, pages 246-251, 2005.

[7] Alain Greiner and Pierre Guerrier. A generic architecture for on-chip paquets-switched interconnections. In Design, Automation and Test in Europe, 2000.

[8] Raj Jain. The Art of Computer Systems Performance Analysis. John Wiley and Sons, Inc., 1991.

[9] Kanishka Lahiri, Sujit Dey, and Anand Raghunathan. Evaluation of the traffic-performance characteristics of system-on-chip communication architectures. In VLSID '01, page 29. IEEE Computer Society, 2001.

[10] Mirko Loghi, Federico Angiolini, Davide Bertozzi, Luca Benini, and Roberto Zafalon. Analyzing on-chip communication in a mpsoc environment. In DATE 04, page 20752, 2004.

[11] Shankar Mahadevan, Federico Angiolini, Michael Storgaard, Rasmus Grøndahl Olsen, Jens Spars $\varnothing$, and Jan Madsen. A network traffic generator model for fast network-on-chip simulation. In DATE 05, pages 780-785, 2005.

[12] Kihong Park and Walter Willinger, editors. Self-Similar Network Traffic and Performance Evaluation. John Wiley \& Sons, 2000.

[13] Santiago Gonzalez Pestana, Edwin Rijpkema, Andrei Rădulescu, Kees Goossens, and Om Prakash Gangwal. Cost-performance trade-offs in networks on chip: A simulation-based approach. In DATE 04, page 20764, 2004.

[14] Frédéric Pétrot and Pascal Gomez. Lightweight implementation of the posix threads api for an on-chip mips multiprocessor with vci interconnect. In DATE 03 Embedded Software Forum, pages 51-56, 2003.

[15] Antoine Scherrer, Nicolas Larrieu, Philippe Owezarski, Pierre Borgnat, and Patrice Abry. Non gaussian and long memory statistical characterisations for internet traffic with anomalies. Technical report, LIP - ENS Lyon, 2005.

[16] R. Thid, M. Millberg, and A. Jantsch. Evaluating NoC communication backbones with simulation. In 21th IEEE Norchip Conference, Riga, November 2003.

[17] Girish Varatkar and Radu Marculescu. On-chip traffic modeling and synthesis for mpeg2 video applications. IEEE Transactions on Very Large Scale Integration (VLSI) Systems, 12(1):108-119, 2004.

[18] Daniel Wiklund, Sumant Sathe, and Dake Liu. Network on chip simulations for benchmarking. In $I W S O C$, pages 269-274, 2004. 